\title{
An Oceanographic Time Capsule
}

\author{
By Walter Munk and Peter Worcester
}

$\mathrm{I}_{\mathrm{N}}$ tained a tidal pressure record on the deep sea floor off California (see Fig. 1 for locations), using a Bourdon-tube pressure sensor and a time-release device for recovery (Filloux, 1969). In summer 1968, Frank Snodgrass dropped capsules Josie, Kathy and Flicki with Vibrotron pressure gauges; the capsules were acoustically recalled after a month (we believe this was the first time this technique was used). These four records, together with a Lamont station 100 miles offshore to the north and cable-connected to shore (Nowroozi et al. 1966) provided the information required for constructing a rough co-tidal chart (Munk et al., 1969).

The outstanding feature in the constructed $M_{2}$ tidal chart was the evidence for an amphidrome roughly midway between San Diego and Hawaii. Stations at opposite sides of an amphidrome must be opposite in phase, and it seemed like a good idea to drop some capsules across the amphidrome to test this simple concept. According to Irish et al. (1971): "In late 1970 we placed three deep-sea instrumented capsules (Kathy, Flicki and Josie) around the predicted amphidrome, using new lownoise quartz-crystal pressure sensors. To this date, only one capsule (Josie) has been recovered...." By 1973, one to two month drops were routinely made as part of the MODE project. When asked: "What was the longest time you ever had a capsule on the sea bottom?" Munk replied "Three years, and getting longer by the year."

The location of Josie (drop) II is shown on the figure; we could not find firm records for the drop locations of Flicki $I I$ and Kathy II but believe they were near the positions shown by the open circles.

Walter Munk, University of California, San Diego, Institute of Geophysics and Planetary Physics, A025, La Jolla, California, 92093; and Peter Worcester, University of California, San Diego. Scripps Institute of Oceanography, A-030, La Jolla, Califomia, 92093.

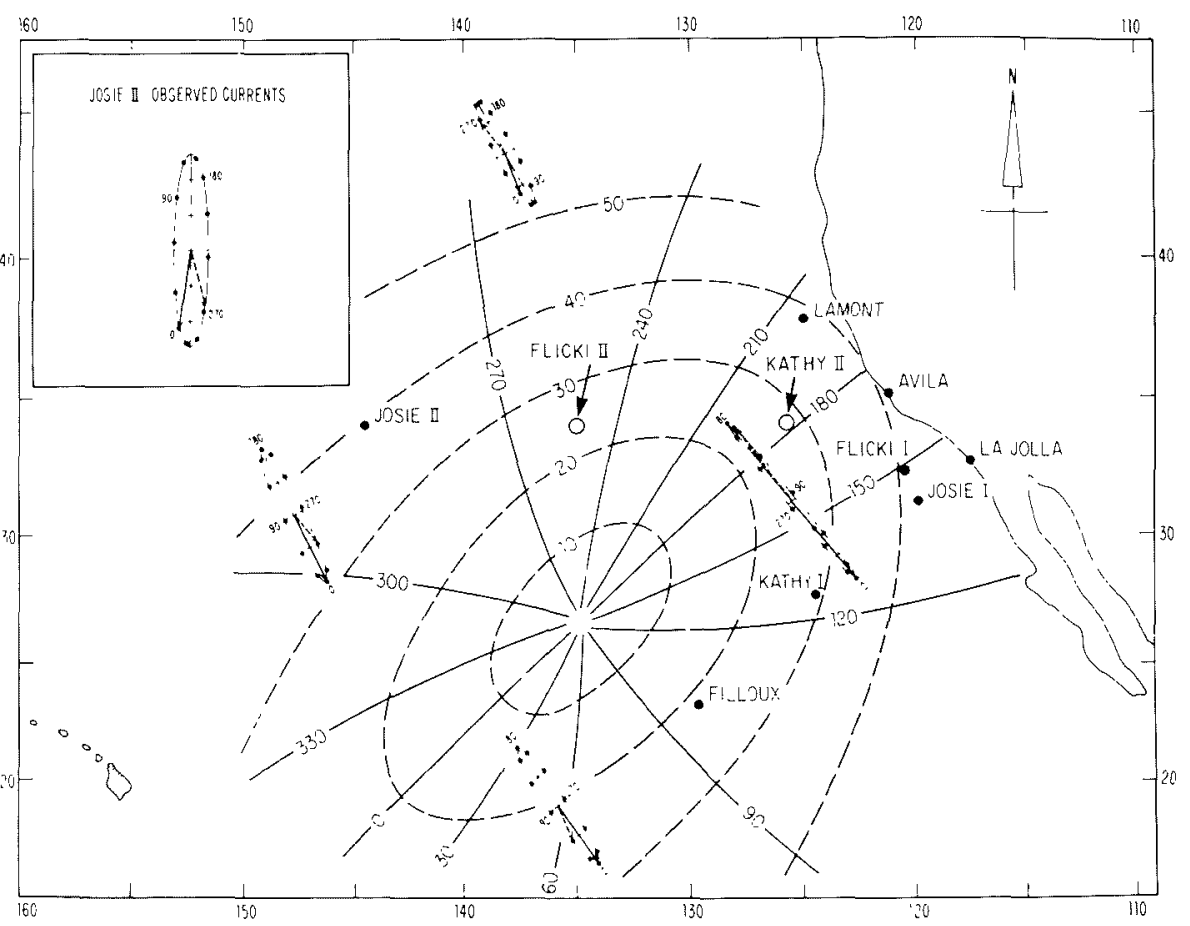

Fig. 1: M, cotidal chart, adapted from Irish et al. (1971). The deep sea capsules Josie, Flicki, and Kathy were dropped at location designated by (I) in 1968 and by (II) in 1970. For the 1970 drop only Josie was recovered; the indicated drop locations for Flicki II and Kathy II are based on memory and are very uncertain.

In September 1988, we received a message from Coast Guard Chief Glen Martineau that a capsule had washed ashore near the Loran $\mathrm{C}$ station at Kure Island in the Midway Island Group. This was 18 years and 2250 nautical miles since the last contact. The capsule was plainly marked "notify Frank Snodgrass, University of California, La Jolla, California." Since Frank died three years ago, this message reached us. Flicki arrived back in San Diego in December 1988 in the best of shape (Fig. 2, p. 49).

Apparently Flicki did not release its anchor when commanded, and remained on the sea floor until the release mechanism or the electromechanical cable connecting the capsule to its sensor package and anchor weight on the sea floor corroded away. At least initially it must have been functioning normally, as the data tape had moved from its original position. It then came to the surface and drifted in the North Pacific until found on Kure Island.

The electronics in the instrument are a remarkable preserved example of antique technology; the interior showed no evidence of leakage. Only a few integrated circuits were used (in the tape recorder circuits). Flip-flop circuits were constructed from discrete components and potted on carriers that could be mounted to circuit cards; "and" gates were constructed from discrete diodes. The data were recorded on 


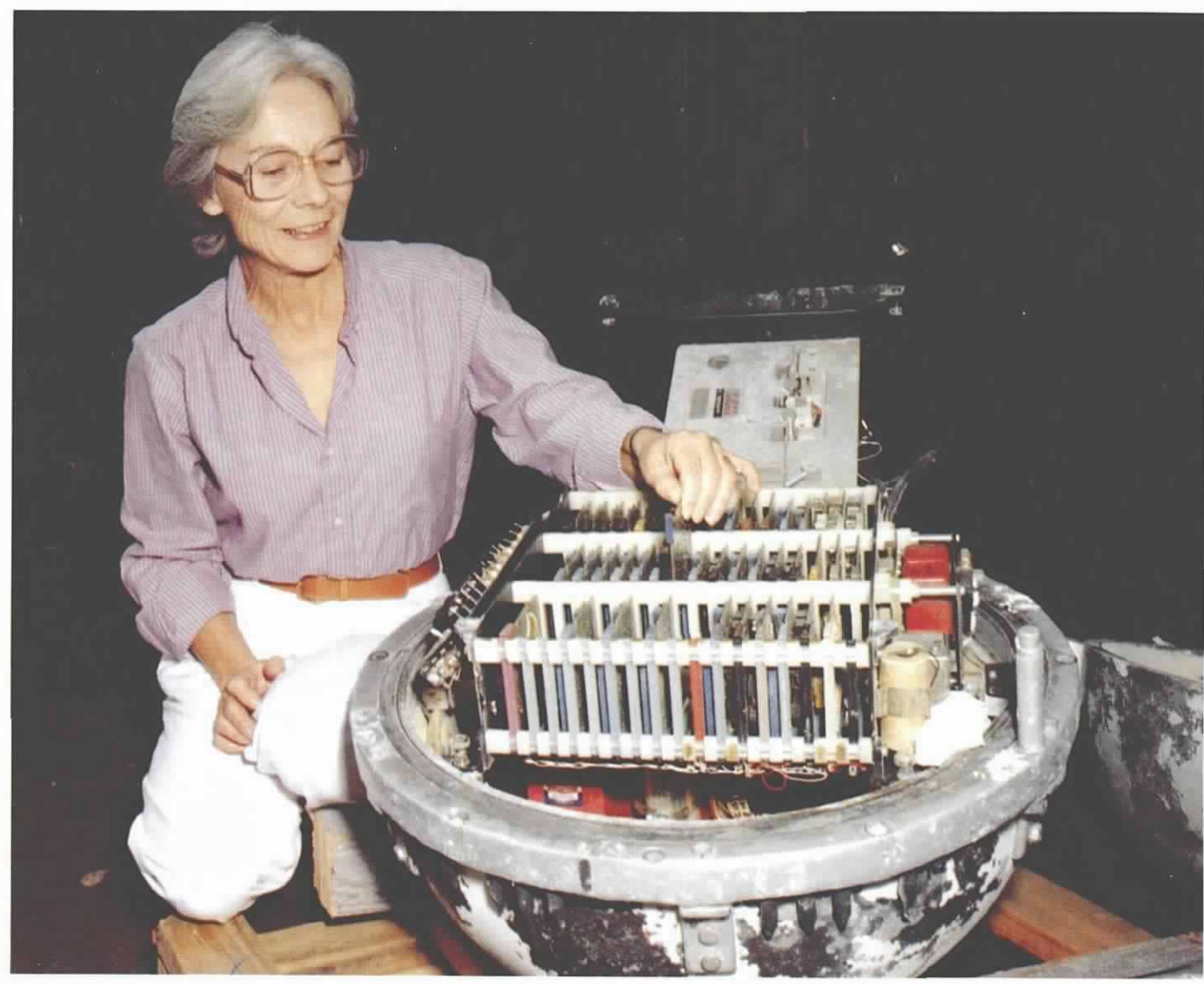

Fig. 2: Flicki Oglebay Dormer (left) and capsule Flicki (right) upon recovery in December 1988. Flicki Oglebay was a programmer at Scripps Institution of Oceanography from 1960 to 1987, and is co-author of the BOMM (for Bullard, Oglebay, Munk and Miller) system of programs for the analysis of time series.

7-track, 200 bpi computer tape that had been loaded into a special cartridge, and had to be manually wound onto a standard computer reel for reading. The tape had a capacity of 720,000 6-bit frames. (For comparison, the instruments that we now use to conduct ocean acoustic tomography experiments have an $80 \mathrm{C} 86$ microprocessor with up to 1 Mbyte of memory as the central controller, roughly equivalent to an IBM PC/XT personal computer, with approximately 20 single-chip microprocessors scattered through the remainder of the instrument to perform various tasks. Data are stored on four 40-Mbyte Winchester disks of the type used in lap-top computers, giving a total of 160 Mbytes of data capacity.)

While the electronics have changed greatly, upon rereading a description of the capsule written 20 years ago (Snodgrass, 1968), we were struck by how up-to-date the remainder of the capsule was. The precision of the quartz crystal pressure and temperature sensors that were used is unsurpassed to this day. The same design of spherical aluminum pressure housings is still in use for ocean bottom seismometers fabricated at Scripps. The capsule had an acoustic command/telemetry system not unlike those currently in use on some subsurface instruments. The basic design of the acoustic release system is similar to ones currently in use, although the electronic components used to implement the filters, etc. have changed greatly. The entire system consumed about 0.25 watts of power, comparable to the power drain of the electronics used for ocean acoustic tomography.

Flicki is truly an oceanographic time capsule. And Kathy is still out there. The answer to the question of "What was the longest time you ever had a capsule on the bottom?" is now 18 years, and getting longer by the year.

\section{References}

Filloux, J. H., 1969: Bourdon Tube Deep-Sea Tide Gages. Proc. Symp. on Tsunamis and Tsunami Research. Honolulu.

Irish, J., W. Munk and F. Snodgrass, 1971: M, Amphidroma in the Northwest Pacific. Geophys. Fluid Dynamics, 2, 355-360.

Munk, W. H., F. Snodgrass and A. M. Wimbush, 1970: Tides Off-shore; Transition from California Coastal to Deep-Sea Waters. Geophys. Fluid Dynamics, I, 161-235.

Nowroozi, A. A., G. H. Sutton and B. Auld, 1966: Oceanic Tides Recorded on the Sea Floor. Ann. Geophys., 22, 512-517.

Snodgrass, F., 1968: Deep Sea Instrument Capsule. Science, $162,78-87 . \square$

\section{A REPORT FROM}

THE INTERNATIONAL

\section{Conference On Tidal}

\section{HYDRODYNAMICS}

\section{By Bruce B. Parker}

\section{T}

1 HE International Conference On Tidal Hydrodynamics was held November 1518,1988 , at the National Institute of Standards and Technology in Gaithersburg, Maryland, USA. It was sponsored by the Office of Oceanography and Marine Assessment in the National Ocean Service, NOAA and the Physical Oceanography Committee of the Marine Technology Society. The conference was attended by 161 tidal researchers from 15 countries. A total of 52 oral papers were presented, plus 21 poster papers.

The conference was divided into six sessions: 1) Tidal Analysis and Prediction; 2) Tidal Hydrodynamic Phenomena and Modeling (subdivided into ocean, shelf, and estuarine tides); 3) Nonlinear Tidal Interactions In Shallow Water; 4) Internal Tides and Baroclinic Effects; 5) New Approaches To Tidal Data Acquisition; and 6) Tidal Applications, Products, and Services. An important part of the program were the ten review papers, describing our present knowledge in particular areas of tidal hydrodynamics.

David Cartwright, formerly of Bidston Observatory in the United Kingdom and presently at the NASA Goddard Flight Center, gave the keynote address and also presented a review paper on tidal detection from satellites. Others giving review papers included: Gabriel Godin (Mississauga, Ontario, Canada) on the analysis of tides and currents; Michael Parke (JPL, CalTech, Pasadena, CA, USA) on ocean tides; Allan Clarke (Florida State, Tallahassee, FL, USA) on continental shelf tides; David Prandle (Bidston Obs., Merseyside, UK) on tides in estuaries and embayments; Bruce Parker(NOS/NOAA, Rockville, MD, USA) on nonlinear tidal interactions; Christian Le Provost (CNRS, Grenoble, France) on the generation of overtides and compound tides; Paul LeBlond (U. of B.C., Vancouver, $\mathrm{BC}$, Canada) on tidal interaction with nontidal phenomena; Helmuth Sandstrom (Bedford Institute, Dartmouth, Nova Scotia, Canada) on internal tides, and Stephen 